\title{
Magnetic two-dimensional electron gas at the manganite-buffered LaAlO3/SrTiO3 interface
}

R. Zhang, H.; Zhang, Y. ; Zhang, H.; Zhang, J.; Shen, X.; Guan, X. X. ; Chen, Yunzhong; Yu, R. C.; Pryds, Nini; Chen, Y. S.

Total number of authors:

12

Published in:

Physical Review B

Link to article, DOI:

10.1103/PhysRevB.96.195167

Publication date:

2017

Document Version

Publisher's PDF, also known as Version of record

Link back to DTU Orbit

Citation (APA):

R. Zhang, H., Zhang, Y., Zhang, H., Zhang, J., Shen, X., Guan, X. X., Chen, Y., Yu, R. C., Pryds, N., Chen, Y. S., Shen, B. G., \& Sun, J. R. (2017). Magnetic two-dimensional electron gas at the manganite-buffered $\mathrm{LaAlO}_{3} / \mathrm{SrTiO}_{3}$ interface. Physical Review B, 96(19), [195167 ]. https://doi.org/10.1103/PhysRevB.96.195167

\section{General rights}

Copyright and moral rights for the publications made accessible in the public portal are retained by the authors and/or other copyright owners and it is a condition of accessing publications that users recognise and abide by the legal requirements associated with these rights.

- Users may download and print one copy of any publication from the public portal for the purpose of private study or research.

- You may not further distribute the material or use it for any profit-making activity or commercial gain

- You may freely distribute the URL identifying the publication in the public portal 


\title{
Magnetic two-dimensional electron gas at the manganite-buffered $\mathrm{LaAlO}_{3} / \mathrm{SrTiO}_{3}$ interface
}

\author{
H. R. Zhang, ${ }^{1,2}$ Y. Zhang,,${ }^{1,2,3}$ H. Zhang, ${ }^{1,2}$ J. Zhang, ${ }^{1,2}$ X. Shen, ${ }^{1,2}$ X. X. Guan, ${ }^{1,2}$ Y. Z. Chen, ${ }^{3, *}$ R. C. Yu, ${ }^{1,2, \dagger}$ N. Pryds, ${ }^{3}$ \\ Y. S. Chen, ${ }^{1,2}$ B. G. Shen, ${ }^{1,2}$ and J. R. Sun ${ }^{1,2, \ddagger}$ \\ ${ }^{1}$ Beijing National Laboratory for Condensed Matter Physics \& Institute of Physics, Chinese Academy of Sciences, \\ Beijing 100190, People's Republic of China \\ ${ }^{2}$ School of Physical Sciences, University of Chinese Academy of Sciences, Beijing 100049, People's Republic of China \\ ${ }^{3}$ Department of Energy Conversion and Storage, Technical University of Denmark, Ris $\phi$ Campus, 4000 Roskilde, Denmark
}

(Received 15 July 2017; revised manuscript received 11 October 2017; published 29 November 2017)

\begin{abstract}
Fabrication of highly mobile spin-polarized two-dimensional electron gas (2DEG) is crucially important for both fundamental and applied research. Usually, spin polarization appears below $10 \mathrm{~K}$ for the 2DEG of $\mathrm{LaAlO}_{3} / \mathrm{SrTiO}_{3}$ interface, stemming from the magnetic ordering of $\mathrm{Ti}^{3+}$ ions with the mediation of itinerant electrons. Herein, we report a magnetic 2DEG at a $\mathrm{La}_{7 / 8} \mathrm{Sr}_{1 / 8} \mathrm{MnO}_{3}$-buffered $\mathrm{LaAlO}_{3} / \mathrm{SrTiO}_{3}$ interface, which simultaneously shows electrically tunable anomalous Hall effect and high conductivity. The spin-polarized temperature for the $2 \mathrm{DEG}$ is promoted to $30 \mathrm{~K}$ while the mobility remains high. The magnetism likely results from a gradient manganese interdiffusion into $\mathrm{SrTiO}_{3}$. The present work demonstrates the great potential of manganite-buffered $\mathrm{LaAlO}_{3} / \mathrm{SrTiO}_{3}$ interfaces for spintronic applications.
\end{abstract}

DOI: 10.1103/PhysRevB.96.195167

\section{INTRODUCTION}

The two-dimensional electron gas (2DEG) formed at the $\mathrm{LaAlO}_{3} / \mathrm{SrTiO}_{3}$ (LAO/STO) interface has attracted extensive attention in recent years because of its great potential for exploration of novel physics and new generation of electronics. [1-11] Since the 2DEG is confined to an ultrathin layer underneath LAO, it is extremely sensitive to surface/interface states. There are various attempts to improve the performance of the 2DEG via surface/interface engineering. As demonstrated by Huijben et al. [12], the impurity scattering at the conducting interface can be strongly reduced by a $\mathrm{SrCuO}_{2}$ cap layer of a few unit cells (uc) in thickness, which causes an increase of carrier mobility from $1 \times 10^{3}$ to $5 \times 10^{4} \mathrm{~cm}^{2} / \mathrm{Vs}$. Through writing charges on sample surface, alternatively Xie et al. [13] promoted the carrier mobility from $6 \times 10^{3}$ to $2 \times 10^{4} \mathrm{~cm}^{2} / \mathrm{Vs}$. An even stronger effect was produced by interface engineering with manganite buffer layers $[14,15]$. As recently shown by Chen et al. [14], the carrier mobility of the 2DEG at the amorphous-LAO/STO interface can be increased from $10^{2}$ up to $7 \times 10^{4} \mathrm{~cm}^{2} / \mathrm{Vs}$ by the introduction of a 1-uc-thick crystalline $\mathrm{La}_{7 / 8} \mathrm{Sr}_{1 / 8} \mathrm{MnO}_{3}$ (LSMO) spacer, amplified by more than two orders of magnitude.

In addition to high mobility, another strongly desired characteristic for oxide 2DEG is spin polarity, which will allow us to explore the core issues of spintronics for all-oxide devices, such as spin generation, spin transport, and spin-to-charge conversion. Unfortunately, reports on ferromagnetic (FM) 2DEG at oxide interfaces remain controversial. Brinkman et al. [5] first reported the signature of magnetism at the interface of $\mathrm{LaAlO}_{3} / \mathrm{SrTiO}_{3}$. They observed a hysteresis loop in magnetoresistance and Kondo effect in the resistancetemperature relation. Based on the analysis of X-ray magnetic circular dichroism, Lee et al. [16] declared a FM order at $10 \mathrm{~K}$ for the 2DEG of LAO/STO. Through magnetic force microscopy, Bi et al. [17] observed magnetic phases at room temperature. Using the technique of scanning superconducting quantum interference device, authors from different groups have observed dipolelike magnetic patches at the interface of $\mathrm{LaAlO}_{3} / \mathrm{SrTiO}_{3}$ [18-20]. Through macroscopic magnetic measurements, Ariando et al. [21] obtained magnetic loops up to room temperature. However, the above magnetism emerges often when electrons are depleted from the interfaces or the establishment of the magnetism does not require interface conduction. So far, anomalous Hall effect (AHE), which is a fingerprint of the ferromagnetism of $2 \mathrm{DEG}$, is detected only at very low temperatures. The first AHE was reported by Shalom et al. [22] for the 2DEG of LAO/STO at $20 \mathrm{mK}$. Then, a spin-polarized 2DEG below $8 \mathrm{~K}$ was declared by Stornaiuolo et al. [23], who inserted a 1-uc EuTiO 3 spacer between LAO and STO and observed AHE. Through optimizing the density of $\mathrm{Sr}$ and $\mathrm{O}$ vacancies in $\mathrm{NdGaO}_{3} / \mathrm{STO}$, recently Gunkel et al. [24] obtained the highest temperature of $\sim 10 \mathrm{~K}$ for the AHE. Obviously, the magnetic ordering temperature suggested by the AHE is much lower than that expected from the macroscopic [21] and microscopic [17] magnetic measurements. It remains elusive how to enhance the FM order of the 2DEG, and whether the introduction of interface magnetism will deteriorate carrier mobility. Inspired by the idea of modulation doping as demonstrated by Chen et al. [14] and Trier et al. [15], we hereby report that gradient manganese doping to interface, by elaborately controlling the interface diffusion of LSMO-buffered LAO/STO, can result in FM exchange of the metallic interface, without slowing down the mobile electrons. By gate-tuning the coupling between local moments and mobile electrons, we succeeded in obtaining spin-polarized 2DEG with the Hall mobility up to $10^{4} \mathrm{~cm}^{2} / \mathrm{Vs}$. Moreover, the upper-limit temperature for the 2DEG to be FM is raised to $\sim 30 \mathrm{~K}$, threefold as large as that previously recorded.

\section{EXPERIMENTS}

\footnotetext{
*yunc@dtu.dk

†rcyu@iphy.ac.cn

${ }^{\ddagger}$ jrsun@iphy.ac.cn
}

Crystalline LAO/LSMO/STO samples with controllable interface diffusion were grown by pulsed-laser ablation with 
the following procedure: At first, an ultrathin amorphous LSMO layer with a predetermined thickness of $t=1,2$, or $3 \mathrm{~nm}$ was deposited on the $\mathrm{TiO}_{2}$-terminated (001)-STO substrate $\left(5 \times 5 \times 0.5 \mathrm{~mm}^{3}\right)$ at ambient temperature, and then an 8-uc-thick crystalline LAO layer, as monitored by reflected high-energy electron diffraction, was grown after heating substrate up to $650{ }^{\circ} \mathrm{C}$. The pulse fluence is $1.5 \mathrm{~J} / \mathrm{cm}^{2}$ and the repetition rate is $1 \mathrm{~Hz}(\lambda=248 \mathrm{~nm})$. After deposition, the sample was cooled to room temperature at a rate of $10{ }^{\circ} \mathrm{C} / \mathrm{min}$ without changing the deposition oxygen pressure of $10^{-5}$ mbar. As expected, the amorphous LSMO is crystallized during heating. Notably, this procedure enables us to achieve highly conducting interface; even the LSMO buffer layer thickness is thicker than $2 \mathrm{uc}(0.8 \mathrm{~nm})$. If a crystalline LSMO buffer layer is epitaxially grown on STO at the very beginning, the resulting LAO/LSMO/STO heterostructures always become insulating when LSMO is thicker than $1 \mathrm{uc}$. To enhance the magnetic effect of LSMO on 2DEG, here we grew a thick amorphous buffer layer rather than a thin crystalline layer. We give a primary explanation for the different electronic behaviors of these two kinds of 2DEGs later. As a reference, a crystalline LAO/STO sample without LSMO buffer was also prepared following the same procedure.

Lattice structure and interfacial chemical composition of the sample were analyzed by an aberration-corrected scanning transmission electron microscope (STEM) with double $\mathrm{C}_{\mathrm{S}}$ correctors (JEM-ARM200F) and an electron energy-loss spectroscopy (EELS), respectively. Resistive measurements were conducted by a Quantum Design physical property measurement system. The van der Pauw geometry was adopted for the measurement and ultrasonic $\mathrm{Al}$ wire bonding was used for electric contact. To tune carrier density, a gate bias was applied at the backside of STO through a Ag electrode while the LAO/LSMO/STO interface was grounded. The maximal leakage current was about $10 \mathrm{nA}$, much lower than the applied current of $10 \mu \mathrm{A}$ for resistance measurements.

\section{RESULTS AND DISCUSSION}

The sharp interface of amorphous-LAO/LSMO(1-uc)/STO is highly conductive but exhibits a linear Hall effect across the whole temperature range from 2 to $300 \mathrm{~K}[14,15]$, indicative of a nonmagnetic state. Now we fabricated our crystalline LAO/LSMO/STO samples with controllable interface diffusion that results in gradient manganese doping to STO by pulsed-laser ablation with the procedure described above. Figure 1(a) is a sketch of the 2DEG at the LAO/LSMO/STO interface, showing an ultrathin LSMO layer sandwiched between nonpolar STO and polar LAO. Analogy to its amorphous counterpart [14], the metallicity of the 2DEG is remarkably improved by the introduction of a LSMO spacer with appropriate thickness $(t)$. As shown in Fig. 1(b), the $R_{\mathrm{S}}(300 \mathrm{~K}) / R_{\mathrm{S}}(2 \mathrm{~K})$ ratio exhibits a more than one order enhancement, varying from $\sim 14$ for $t=0$ to $\sim 186$ for $t=$ $1 \mathrm{~nm}$. When $t$ exceeds $2 \mathrm{~nm}$, however, the sample turns out to be highly insulating (see Supplemental Material [25]). These results indicate that a thick LSMO spacer has blocked the polar-discontinuity-induced charge transfer from LAO to STO. According to the polar catastrophe model, half an electron per unit cell will be transferred from the surface $\mathrm{AlO}_{2}$ layer to (a)

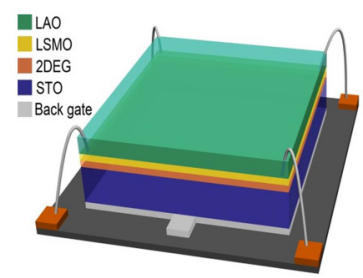

(c)

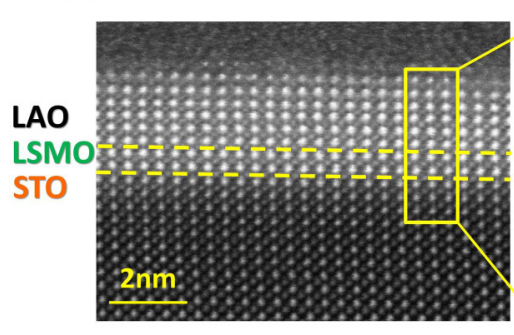

(b)

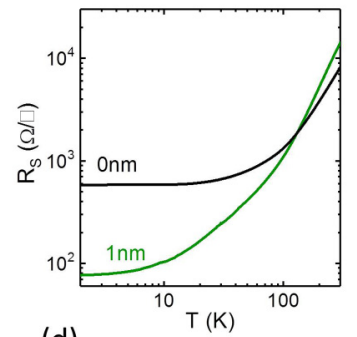

(d)

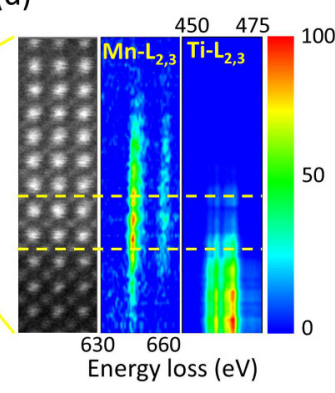

FIG. 1. (a) Sketch of the 2DEG at the LAO/LSMO/STO interface. (b) Temperature dependence of the sheet resistance for LAO/STO and $\mathrm{LAO} / \mathrm{LSMO} / \mathrm{STO}$ with LSMO spacer of $1 \mathrm{~nm}$. In incorporation of 1-nm LSMO considerably improves the metallicity of the 2DEG despite interlayer diffusion. (c) HAADF image of the cross section of the sample. The manganite buffer layer is marked by dashed lines. (d) EELS mappings across the Mn- $L_{2,3}$ and Ti- $L_{2,3}$ edges, corresponding to the enlarged HAADF image shown in the left column. Dashed lines mark interfaces.

the LAO/STO interface. However, if a LSMO buffer layer exists between LAO and STO, electron from LAO will first fill into the Mn $e_{g}$ states of LSMO since they exhibit lower energy levels than the Ti $t_{2 g}$ states [14]. When the LSMO layer is thick enough, it will completely prevent the LAO to STO charge transfer. Hereafter, we will focus on the optimal sample of LAO/LSMO(1-nm)/STO.

Figure 1(c) presents the high-angle annular dark-field (HAADF) image of the cross section of LAO/LSMO(1nm)/STO. The bright strips are heavy $A$-site atoms of the perovskite structure ( $\mathrm{La}$ and $\mathrm{Sr}$ ). $B$-site atoms (Ti, Mn, and Al) are light compared with the $A$-site ones, forming slightly dim strips. A clear LSMO/STO interface can be identified due to the sharp La to Sr contrast (marked by a yellow dashed line). Remarkably, the originally amorphous LSMO layer has been turned into crystalline after the high-temperature growth process of LAO, forming a high degree of epitaxy. Due to the similar contrast of $\mathrm{Mn}$ and $\mathrm{Al}$, the $\mathrm{LAO} / \mathrm{LSMO}$ interface is not clear in the HAADF image. According to the EELS analysis, the layer thickness of LSMO is about 2 uc, close to the preset $1 \mathrm{~nm}$. The EELS mappings across the Mn- $L_{2,3}$ and Ti- $L_{2,3}$ edges reveal a LSMO/STO interlayer diffusion [Fig. 1(d)]: Considerable $\mathrm{Mn}$ and $\mathrm{Ti}$ ions exchange sites in the range of 3-4 unit cells. A quantitative analysis of the EELS spectra shows that the concentration of $\mathrm{Mn}$ is $\sim 24 \%, \sim 9 \%, \sim 6 \%$, $\sim 1 \%$, and null in the first to fifth $\mathrm{TiO}_{2}$ layer, i.e., a gradient doping of Mn into STO has taken place. Additionally, obvious Mn upward diffusion across the LAO/LSMO interface also 

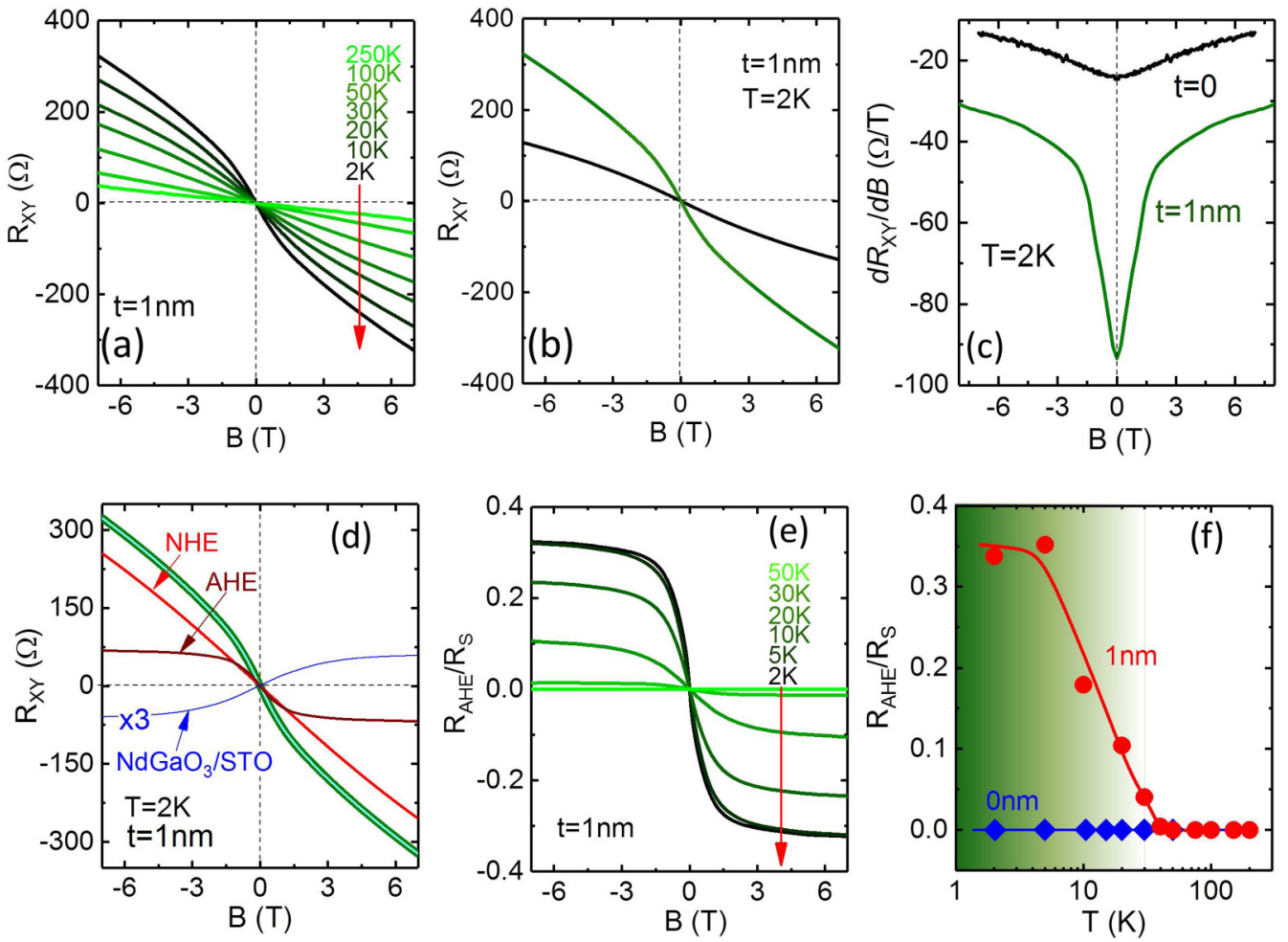

FIG. 2. (a) Magnetic field dependence of the Hall resistance $\left(R_{X Y}\right)$ for the LAO/LSMO $(1-\mathrm{nm}) / \mathrm{STO}$ interface, measured in the temperature from 2 to $250 \mathrm{~K}$. (b), (c) Comparison of the Hall effect for two metallic interfaces of $t=0$ and $1 \mathrm{~nm}$, measured at $T=2 \mathrm{~K}$, and the corresponding Hall coefficient. (d) Example for the determination of NHE and AHE from the total Hall effect at $2 \mathrm{~K}$. Measured and calculated results are presented as thick green and thin cyan lines, respectively. Blue line marks the AHE of $\mathrm{NdGaO}_{3} / \mathrm{STO}$ (Ref. [24]). (e) Deduced AHE as a function of magnetic field at different temperatures. (f) Normalized anomalous Hall resistance (at $B=7 \mathrm{~T}$ ) as a function of temperature for $t=0$ and $1 \mathrm{~nm}$.

takes place [Fig. 1(d)]. However, this will not affect the 2DEG at the LSMO/STO interface.

Our following magnetotransport measurement reveals the presence of a nonlinear Hall effect in the LSMO-buffered sample of $t=1 \mathrm{~nm}$. Summarized in Fig. 2(a) is the Hall resistance $\left(R_{X Y}\right)$ of $\mathrm{LAO} / \mathrm{LSMO}(1-\mathrm{nm}) / \mathrm{STO}$, measured in the temperature range from 2 to $250 \mathrm{~K}$ as a function of magnetic field $(B)$. When temperature is high, $R_{X Y}$ varies linearly with applied field. This is the typical behavior of the normal Hall effect (NHE), and gives rise to a carrier density of $\sim 1.4 \times 10^{14} \mathrm{~cm}^{-2}$ at $295 \mathrm{~K}$ and $\sim 5.3 \times 10^{13} \mathrm{~cm}^{-2}$ at $75 \mathrm{~K}$. When the sample is cooled to $50 \mathrm{~K}$, curve bending emerges around $B=0$ and develops upon further cooling to $2 \mathrm{~K}$. Nonlinear Hall effect is also observed in unbuffered LAO/STO interface [Fig. 2(b)]. But, it can be described by a two-band model as previously reported by Joshua et al. [26]. It is therefore a NHE in nature. In contrast, our buffered sample $(t=1 \mathrm{~nm})$ exhibits a much stronger curvature in the low-field range $(|B|<1.7 \mathrm{~T})$, which cannot be reproduced by the two-band model. To highlight this feature, in Fig. 2(c) we show the Hall coefficient defined by the differential of $R_{X Y}$ with respect to $B$. Different from the broad concave around $B=0$ of the unbuffered LAO/STO, the Hall coefficient of LAO/LSMO(1-nm)/STO forms a deep pit. Instead, as illustrated in Fig. 2(d), this nonlinear Hall effect for $t=1 \mathrm{~nm}$ can only be reproduced by an extended two-band model that combines the two-band conduction-dominated NHE with an
AHE effect (see Supplemental Material [25]),

$$
R_{\mathrm{HE}}=R_{\mathrm{NHE}}(\text { two band })+\alpha L\left(m B / k_{\mathrm{B}} T\right) .
$$

Here, the Langevin function $L(x)$ is introduced to simulate step-shaped AHE curve in form, $\alpha$ is a scale factor, and $m$ is magnetic moment (see Supplemental Material [25] for two-band model calculation). As illustrated in Fig. 2(d), the model calculation (thin cyan line) well reproduces the measured (thick green line) Hall resistance (the deduced carrier densities and mobilities are shown in Fig. 4). Basically, the normal Hall resistance $\left(R_{\mathrm{NHE}}\right)$ varies smoothly with $H$ in the whole field range, with slight but identifiable curve bending. In contrast, the anomalous Hall resistance $\left(R_{\mathrm{AHE}}\right)$ is constant in high-field range and undergoes a drastic change as $B$ sweeps through a narrow-field range around $B=0$. Notably, $R_{\mathrm{AHE}}$ exhibits an opposite sign to magnetic field. It is this feature that leads to the deep pit of the Hall coefficient. This is in sharp contrast to $\mathrm{NdGaO}_{3} / \mathrm{STO}$ [24] [blue curves in Fig. 2(d)] or $\mathrm{LAO} / \mathrm{EuTiO}_{3} / \mathrm{STO}$ [23], for which $R_{\mathrm{AHE}}$ owns the same sign as the applied field. The change in the AHE sign may result from the difference in spin polarization of our buffered sample.

The Hall resistance displayed in Fig. 2(d) for the $\mathrm{NdGaO}_{3} / \mathrm{STO}$ interface comes from Ref. [24]. It is the anomalous Hall resistance. The corresponding data were extracted from the $R_{X Y}-B$ curve at $2 \mathrm{~K}$ in Fig. 2(a) of 
Ref. [24]. The normal and anomalous Hall resistances have been determined by the technique described there.

Figure 2(e) shows the deduced $R_{\mathrm{AHE}}-B$ dependence as a function of temperature, where $R_{\mathrm{AHE}}$ has been normalized by $R_{\mathrm{S}}$ to compare with the results of different samples. All of the $R_{\mathrm{AHE}}-B$ curves are step-shaped, with the most drastic changes taking place between -2 and $2 \mathrm{~T}$. With the increase of temperature, the step height decreases first slowly and then rapidly. However, the general $R_{\mathrm{AHE}}-B$ dependence remains unchanged, i.e., the saturation field for $R_{\mathrm{AHE}}$ does not vary with temperature. As a summary, in Fig. 2(f) we show the temperature dependence of the normalized $R_{\mathrm{AHE}}$, recorded under a field of $7 \mathrm{~T}$. No AHE is visible for the sample of $\mathrm{LAO} / \mathrm{STO}$ even in the low-temperature regime. For the sample of $t=1 \mathrm{~nm}$, AHE sets in at $30 \mathrm{~K}$, develops rapidly upon further cooling, and saturates below $5 \mathrm{~K}$. We have prepared three $t=1 \mathrm{~nm}$ samples with the same condition, and observed essentially the same electronic behaviors.

The LSMO buffer layer is insulating (not shown), and its resistance is well above that of 2DEG. As a result, when the LSMO buffer layer and the 2DEG are measured simultaneously, the Hall signals are mainly contributed by the 2DEG, i.e., the observed $R_{\mathrm{ANE}}$ comes from the buffered 2DEG. In general, AHE stems from the asymmetric scattering of charge carriers from orientated magnetization [27]. Its appearance implies the occurrence of spin polarization and long-range FM order in our buffered sample. Notably, compared to the $\mathrm{NdGaO}_{3}$ (8-uc)/STO [24] and $\mathrm{LAO} / \mathrm{EuTiO}_{3} / \mathrm{STO}$ [23], where the AHE occurs at a temperature below $8 \mathrm{~K}$, the temperature span for our 2DEG to be spin-polarized has been threefold expanded $(\sim 30 \mathrm{~K})$. As will be seen later, this could be ascribed to the gradient doping of Mn to STO.

In addition to temperature, gate biases $\left(V_{\mathrm{G}}\right)$ also have a strong effect on AHE. As shown in Fig. 3(a), positive $V_{\mathrm{G}}$ bends the $R_{X Y}-B$ curve further, whereas the negative ones flatten it. The gate dependence of the $R_{\mathrm{AHE}}$ is given in Fig. 3(b), deduced from a quantitative analysis of the $R_{X Y}-B$ curve. The maximal $R_{\mathrm{AHE}} / R_{\mathrm{S}}$ is $\sim 1.2 \Omega$, appearing under an applied voltage of 40 $\mathrm{V}$. It decreases as $V_{\mathrm{G}}$ sweeps from 40 to $-20 \mathrm{~V}$, and completely vanishes below $-30 \mathrm{~V}$. Accompanying the weakening of the AHE, the Kondo effect develops [Fig. 3(c)]. These features are similar to those of the $2 \mathrm{DEG}$ of $\mathrm{LAO} / \mathrm{EuTiO}_{3} / \mathrm{STO}$ but the local magnetic moment now is from $\mathrm{Mn}^{2+}$ or $\mathrm{Mn}^{3+}$ rather than $\mathrm{Ti}^{3+}$. It should be emphasized that our AHE is distinct in a sense it shows an opposite sign to the reported one $[23,24]$. This implies a change in the manner of spin scattering when a LSMO spacer is inserted between LAO and STO. Probably, besides the $\mathrm{Ti}^{3+}$ scattering centers as reported in

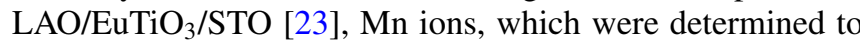
be $\mathrm{Mn}^{2+}$ according to the x-ray photoelectron spectroscopy analysis (see Supplemental Material [25]), also contribute to magnetic scattering. This conclusion seems to be supported by a quantitative analysis of the Kondo effect. As shown by the solid line in Fig. 3(c), the resistance upturn can be well reproduced by the standard formula [28-30]

$$
R=R_{0}+a T^{b}+R_{k}\left(\frac{1}{1+\left(2^{1 / s}-1\right)\left(T / T_{k}\right)^{2}}\right)^{s},
$$

with the parameters of $R_{0}=21.4 \pm 0.2 \Omega / \square, R_{k}=40.0 \pm$ $0.5 \Omega / \square, T_{k}=13.4 \pm 0.2 \mathrm{~K}, a=0.38 \pm 0.02 \Omega / \square \mathrm{K}^{1.42}, b=$ $1.42 \pm 0.01$, and $S=1.1 \pm 0.2$. A clear Kondo effect is also observed under the $V_{\mathrm{G}}$ of -30 and $-40 \mathrm{~V}$, and well described by Eq. (2) adopting appropriate fitting parameters (see Supplemental Material [25]). A common feature of these fitting parameters is that the $S$ is close to 1.1. Notably, $S=1.1$ is much larger than the 0.225 that is usually obtained for a $\mathrm{Ti}^{3+}$ ion [30]. Considering Kondo screening, this result means the involvement of additional ions in the process of Kondo scattering, with a spin well above 1.1. This result echoes the observation of significant diffusion of Mn ions into STO. According to the EELS analysis, gradient doping of $\mathrm{Mn}$ into STO has taken place. The Mn content in the first $\mathrm{TiO}_{2}$ layer can be as high as $\sim 24 \%$. It corresponds to a density of $\sim 1.6 \times 10^{14} \mathrm{~cm}^{-2}$, i.e., most of the electrons that are expected to be transferred to the interface within the polar catastrophe model are now localized by Mn ions. As spin scattering centers, obviously $\mathrm{Mn}^{2+} / \mathrm{Mn}^{3+}$ could be much more effective than $\mathrm{Ti}^{3+}$ because of its large magnetic moment $\left(4 \mu_{\mathrm{B}} / \mathrm{Mn}^{3+}, 5 \mu_{\mathrm{B}} / \mathrm{Mn}^{2+}\right.$, and $\left.1 \mu_{\mathrm{B}} / \mathrm{Ti}^{3+}\right)$.

Figures 4(a)-4(d) summarize the carrier density and mobility as functions of temperature and gate field. As shown in Figs. 4(a) and 4(b), there are two species of charge carriers in the 2DEG at low temperatures, a slow one and a fast one. With the increase of temperature, the slow carrier density $\left(n_{1}\right)$ exhibits a monotonic growth whereas the fast one $\left(n_{2}\right)$
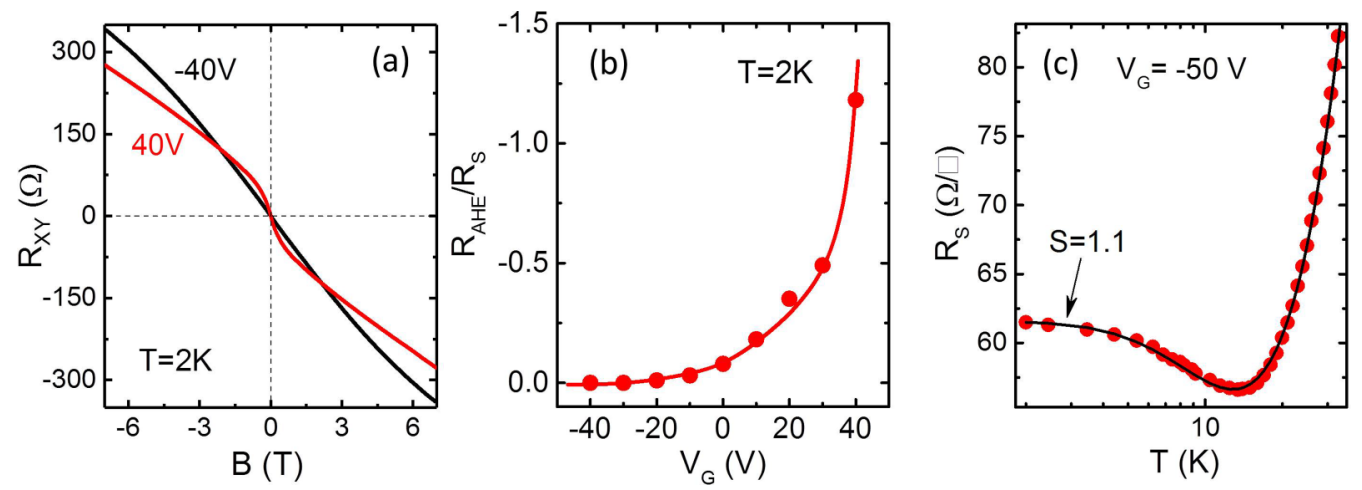

FIG. 3. (a) Hall resistance of the LAO/LSMO(1-nm)/STO samples, measured under different gate biases at $2 \mathrm{~K}$. (b) Deduced anomalous Hall resistance as a function of gate voltage obtained at $2 \mathrm{~K}$. Solid line is a guide for the eye. (c) Kondo effect recorded under a gate voltage of $-50 \mathrm{~V}$ (symbols). Solid lines are calculated results based on Eq. (2), evidencing the presence of magnetic ions other than $\mathrm{Ti}^{3+}$. 

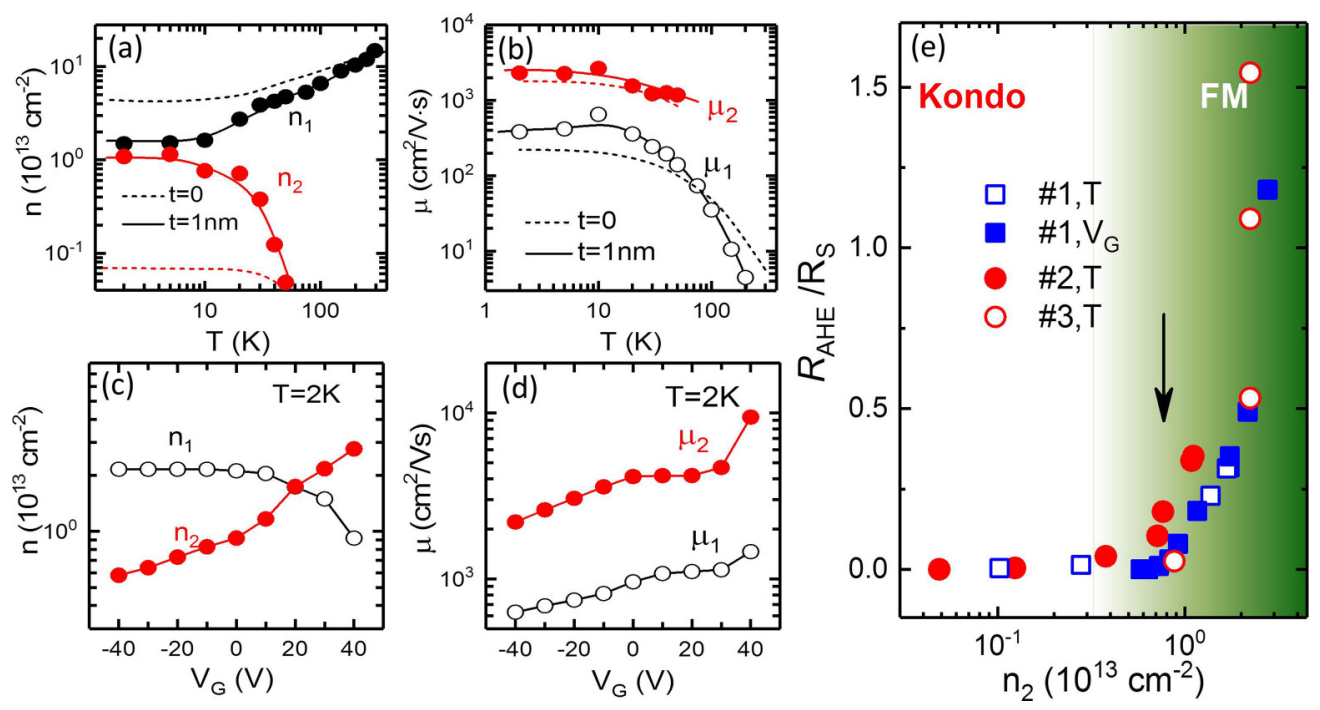

FIG. 4. (a),(b) Carrier density and mobility, respectively, as a function of temperature, determined by the two-band model based on the Hall effect. Two species of charge carrier coexist below $50 \mathrm{~K}$. Dashed lines are carrier density (a) and mobility (b) for the sample of $t=0$. (c),(d) Carrier density and mobility as a function of gate voltage, respectively, recorded at $2 \mathrm{~K}$. (e) Normalized anomalous Hall resistance as a function of the density of fast charge carriers. Numbers in the figure denote three different samples with the same LSMO spacer of 1 nm. " $T$ " or " $V_{\mathrm{G}}$ " represent the data obtained by tuning temperature or gate voltage. Arrow marks the threshold carrier density for the AHE.

decreases first slowly and then rapidly, and finally vanishes above $50 \mathrm{~K}$. Fixing temperature to $2 \mathrm{~K}$ while ramping the gate voltage from -40 to $+40 \mathrm{~V}, n_{1}$ is decreased from $2.2 \times 10^{13}$ to $0.9 \times 10^{13} \mathrm{~cm}^{-2}$ and $n_{2}$ is increased from $0.6 \times 10^{13}$ to $2.7 \times 10^{13} \mathrm{~cm}^{-2}$ [Figs. 4(c) and 4(d)]. According to the work of Smink et al. [31], an electron-electron interaction term will cause an upward shift of the $d_{X Y}$ subbands when the $d_{Y Z} / d_{X Z}$ subbands become occupied, resulting in a spilling from the $d_{X Y}$ to the $d_{Y Z} / d_{X Z}$ subbands. This may explain the decrease in $n_{1}$ and the concomitant increase in $n_{2}$ when $V_{\mathrm{G}}$ exceeds $\sim 15 \mathrm{~V}$ [Fig. 4(c)]. Interestingly, the heavy manganese doping does not produce any adverse effect on mobility, probably due to gradient distribution in STO. As a comparison, $n_{1}$ and $n_{2}$ of the LAO/STO interface are also deduced, and are shown as dashed lines in Fig. 4(a). $n_{1}$ is on the order of $10^{13} \mathrm{~cm}^{-2}$ while $n_{2}$ is only $\sim 7 \times 10^{11} \mathrm{~cm}^{-2}$.

Presumably, the fast and slow charge carriers may be mainly the $d_{Y Z} / d_{Z X}$ and $d_{X Y}$, electrons, respectively. As well documented [32,33], the $d_{X Y}$ electrons have a larger population at interfacial layers, but their mobility is rather low, probably due to strong interfacial scattering despite their light effective mass. In contrast, $d_{X Z} / d_{Y Z}$ electrons exhibit a much lower carrier density than the $d_{X Y}$ ones, which occupy the inner $\mathrm{TiO}_{2}$ layers, and exhibit a relatively high mobility.

A close inspection of the above data is suggestive. Based on both the temperature and the gate-bias dependence, the Kondo effect shows up whereas the AHE vanishes when the density of slow charge carriers $n_{1}$ is much larger than that of fast ones $n_{2}$. In the opposite situation, however, the AHE emerges but the Kondo behavior disappears. Obviously, the slow and fast charge carriers interact with magnetic moments in a different manner. The former causes a Kondo screening, whereas the latter produce a magnetic coupling between local moments. It is their competition that determines the transport property of the 2DEG: The $d_{X Y}$ electrons suffer from the magnetic scattering of $\mathrm{Mn}$ ions, resulting in the resistance upturn below $\sim 14 \mathrm{~K}$. However, when the density of $d_{X Z} / d_{Y Z}$ electrons is high enough, the FM exchange between Mn ions prevails, overwhelming the Kondo scattering. This is consistent with the declaration that the $d_{X Z} / d_{Y Z}$ electrons can mediate a FM exchange between local moments since they disperse substantially in the $z$ direction whereas the $d_{X Y}$ electrons does not due to their 2D character [23].

To get a clear picture on the effect of fast charge carriers, in Fig. 4(e) we show the $R_{\mathrm{AHE}}$ to $n_{2}$ relation for three different LAO/LSMO(1-nm)/STO samples, obtained by tuning $T$ from 2 to $50 \mathrm{~K}$ but keeping $V_{\mathrm{G}}=0$ or sweeping $V_{\mathrm{G}}$ from -40 to $40 \mathrm{~V}$ while keeping $T=2 \mathrm{~K}$. In the low- $n_{2}$ regime, the Kondo effect dominates the transport process. However, it gives its way to AHE above the carrier density of $n_{2}=7 \times 10^{12} \mathrm{~cm}^{-2}$, irrespective of the ways that tune $n_{2}$. For the $t=0$ sample, carrier density of $n_{2}$ is very low, $\sim 7 \times 10^{11} \mathrm{~cm}^{-2}$. Therefore, no FM order is established.

We have tried to insert a crystalline LSMO buffer layer into the LAO/STO interface, and found that the 2DEG always disappeared when layer thickness exceeded $1 \mathrm{uc}$, regardless of the growth temperature of the LAO top layer. It is an interesting question why the electronic behavior of the 2DEGs is so different when buffered by a recrystallized amorphous-LSMO layer and an initially crystalline LSMO layer. There are signatures that the crystallization of the amorphous layer has caused unique processes. First, a deep doping of manganese into STO has taken place. According to the STEM analysis [Fig. 1(d)], Mn ions have diffused into STO by 4 uc in the deposition process of the LAO cap layer, whereas they are only 1 uc into STO when a crystalline LSMO layer is directly deposited on STO [34]. The deep manganese doping will be helpful for the building up of a magnetic 2DEG. Second, substantial site exchange took place between $\mathrm{Mn}$ and $\mathrm{Al}$ around the LAO/LSMO interface [Fig. 1(d)]. This 
means that the separation between some $\mathrm{AlO}_{2}$ layers and STO could be shorter than 2 uc. As a result, the blocking effect of the LSMO layer on LAO-STO charge transfer is partially broken. This explains why our 2DEG remains metallic when the LSMO layer is thick. Presumably, the Mn-Ti and Mn-Al site exchanges take place accompanying the amorphous-tocrystalline transition of the LSMO layer. However, the detailed processes are still not very clear at present, and require further investigations.

\section{CONCLUSION}

In conclusion, magnetic $2 \mathrm{DEG}$ at the $\mathrm{LAO} / \mathrm{LSMO} / \mathrm{STO}$ interface has been obtained by gradient manganese doping into the interfacial layer of STO. It simultaneously shows an electrically tunable FM order and high carrier mobility. The temperature range for the $2 \mathrm{DEG}$ to be spin polarized is $30 \mathrm{~K}$, threefold as wide as that without magnetic doping. There are indications that the magnetic exchange occurs between doped manganese ions, with the mediation of $d_{X Z} / d_{Y Z}$ electrons. The present work opens an avenue toward high-performance spin-polarized 2DEG.

\section{ACKNOWLEDGMENTS}

This work has been supported by the National Basic Research of China (Grants No. 2016YFA0300701 and No. 2017YFA0206300), the National Natural Science Foundation of China (Grants No. 11520101002, No. 51590880, No. 11374348, No. 11134007, No. 11574376, and No. 11574366), and the Key Program of the Chinese Academy of Sciences.
[1] H. Y. Hwang, Y. Iwasa, M. Kawasaki, B. Keimer, N. Nagaosa, and Y. Tokura, Nat. Mater. 11, 103 (2012).

[2] A. D. Caviglia, S. Gariglio, C. Cancellieri, B. Sacépé, A. Fête, N. Reyren, M. Gabay, A. F. Morpurgo, and J.-M. Triscone, Phys. Rev. Lett. 105, 236802 (2010).

[3] N. Reyren, S. Thiel, A. D. Caviglia, L. Fitting Kourkoutis, G. Hammerl, C. Richter, C. W. Schneider, T. Kopp, A.-S. Rüetschi, D. Jaccard, M. Gabay, D. A. Muller, J.-M. Triscone, and J. Mannhart, Science 317, 1196 (2007).

[4] A. D. Caviglia, S. Gariglio, N. Reyren, D. Jaccard, T. Schneider, M. Gabay, S. Thiel, G. Hammerl, J. Mannhart, and J.-M. Triscone, Nature (London) 456, 624 (2008).

[5] A. Brinkman, M. Huijben, M. van Zalk, J. Huijben, U. Zeitler, J. C. Maan, W. G. van der Wiel, G. Rijnders, D. H. A. Blank, and H. Hilgenkamp, Nat. Mater. 6, 493 (2007).

[6] A. D. Caviglia, M. Gabay, S. Gariglio, N. Reyren, C. Cancellieri, and J.-M. Triscone, Phys. Rev. Lett. 104, 126803 (2010).

[7] Y. W. Xie, C. Bell, T. Yajima, Y. Hikita, and H. Y. Hwang, Nano Lett. 10, 2588 (2010).

[8] S. Thiel, G. Hammerl, A. Schmehl, C. W. Schneider, and J. Mannhart, Science 313, 1942 (2006).

[9] C. Cen, S. Thiel, G. Hammerl, C. W. Schneider, K. E. Andersen, C. S. Hellberg, J. Mannhart, and J. Levy, Nat. Mater. 7, 298 (2008).

[10] C. Bell, S. Harashima, Y. Kozuka, M. Kim, B. G. Kim, Y. Hikita,and H. Y. Hwang, Phys. Rev. Lett. 103, 226802 (2009).

[11] E. Lesne, Y. Fu, S. Oyarzun, J. C. Rojas-Sánchez, D. C. Vaz, H. Naganuma, G. Sicoli, J.-P. Attané, M. Jamet, E. Jacquet, J.-M. George, A. Barthélémy, H. Jaffrès, A. Fert, M. Bibes, and L. Vila, Nat. Mater. 15, 1261 (2016).

[12] M. Huijben, G. Koster, M. K. Kruize, S. Wenderich, J. Verbeeck, S. Bals, E. Slooten, B. Shi, H. J. A. Molegraaf, J. E. Kleibeuker, S. van Aert, J. B. Goedkoop, A. Brinkman, D. H. A. Blank, M. S. Golden, G. van Tendeloo, H. Hilgenkamp, and G. Rijnders, Adv. Funct. Mater. 23, 5240 (2013).

[13] Y. W. Xie, C. Bell, Y. Hikita, S. Harashima, and H. Y. Hwang, Adv. Mater. 25, 4735 (2013).

[14] Y. Z. Chen, F. Trier, T. Wijnands, R. J. Green, N. Gauquelin, R. Egoavil, D. V. Christensen, G. Koster, M. Huijben, N. Bovet, S. Macke, F. He, R. Sutarto, N. H. Andersen, J. A. Sulpizio,
M. Honig, G. E. D. K. Prawiroatmodjo, T. S. Jespersen, S. Linderoth, S. Ilani, J. Verbeeck, G. Van Tendeloo, G. Rijnders, G. A. Sawatzky, and N. Pryds, Nat. Mater. 14, 801 (2015).

[15] F. Trier, G. E. D. K. Prawiroatmodjo, Z. Zhong, D. V. Christensen, M. von Soosten, A. Bhowmik, J. M. G. Lastra, Y. Chen, T. S. Jespersen, and N. Pryds, Phys. Rev. Lett. 117, 096804 (2016).

[16] J.-S. Lee, Y. W. Xie, H. K. Sato, C. Bell, Y. Hikita, H. Y. Hwang, and C.-C. Kao, Nat. Mater. 12, 703 (2013).

[17] F. Bi, M. C. Huang, S. Ryu, H. Lee, C. W. Bark, C. B. Eom, P. Irvin, and J. Levy, Nat. Commun. 5, 5019 (2015).

[18] J. A. Bert, B. Kalisky, C. Bell, M. Kim, Y. Hikita, H. Y. Hwang, and K. A. Moler, Nat. Phys. 7, 767 (2011).

[19] B. Kalisky, J. A. Bert, B. B. Klopfer, C. Bell, H. K. Sato, M. Hosoda, Y. Hikita, H. Y. Hwang, and K. A. Moler, Nat. Commun. 3, 922 (2012).

[20] B. Kalisky, J. A. Bert, C. Bell, Y. Xie, H. K. Sato, M. Hosoda, Y. Hikita, H. Y. Hwang, and K. A. Moler, Nano Lett. 12, 4055 (2012).

[21] X. Wang Ariando, G. Baskaran, Z. Q. Liu, J. Huijben, J. B. Yi, A. Annadi, A. R. Barman, A. Rusydi, S. Dhar, Y. P. Feng, J. Ding, H. Hilgenkamp, and T. Venkatesan, Nat. Commun. 2, 188 (2011).

[22] M. B. Shalom, M. Sachs, D. Rakhmilevitch, A. Palevski, and Y. Dagan, Phys. Rev. Lett. 104, 126802 (2010).

[23] D. Stornaiuolo, C. Cantoni, G. M. D. Luca, R. D. Capua, E. D. Gennaro, G. Ghiringhelli, B. Jouault, D. Marrè, D. Massarotti, F. M. Granozio, I. Pallecchi, C. Piamonteze, S. Rusponi, F. Tafuri, and M. Salluzzo, Nat. Mater. 15, 278 (2016).

[24] F. Gunkel, C. Bell, H. Inoue, B. Kim, A. G. Swartz, T. A. Merz, Y. Hikita, S. Harashima, H. K. Sato, M. Minohara, and H. Y. Hwang, Phys. Rev. X 6, 031035 (2016).

[25] See Supplemental Material at http://link.aps.org/supplemental/ 10.1103/PhysRevB.96.195167 for the sheet conduction and carrier density of $\mathrm{LaAlO}_{3} / \mathrm{La}_{7 / 8} \mathrm{Sr}_{1 / 8} \mathrm{MnO}_{3} / \mathrm{SrTiO}_{3}$ interfaces, two-band model calculation, $\mathrm{x}$-ray photoemission microscopy of Mn, and Kondo effect. Supplemental Material also includes Refs. [2,15,22,24,32].

[26] A. Joshua, S. Pecker, J. Ruhman, E. Altman, and S. Ilani, Nat. Commun. 3, 1129 (2012). 
[27] N. Naoto Nagaosa, J. Sinova, S. Onoda, A. H. MacDonald, and N. P. Ong, Rev. Mod. Phys. 82, 1539 (2010).

[28] D. Goldhaber-Gordon, J. Göres, M. A. Kastner, H. Shtrikman, D. Mahalu, and U. Meirav, Phys. Rev. Lett. 81, 5225 (1998).

[29] M. Lee, J. R. Williams, S. Zhang, C. D. Frisbie, and D. Goldhaber-Gordon, Phys. Rev. Lett. 107, 256601 (2011).

[30] W. N. Lin, J. F. Ding, S. X. Wu, Y. F. Li, J. Lourembam, S. Shannigrahi, S. J. Wang, and T. Wu, Adv. Mater. Interfaces 1, 1300001 (2014).
[31] A. E. M. Smink, J. C. de Boer, M. P. Stehno, A. Brinkman, W. G. van derWiel, and H. Hilgenkamp, Phys. Rev. Lett. 118, 106401 (2017).

[32] P. Delugas, A. Filippetti, V. Fiorentini, D. I. Bilc, D. Fontaine, and P. Ghosez, Phys. Rev. Lett. 106, 166807 (2011).

[33] S. Banerjee, O. Erten, and M. Randeria, Nat. Phys. 9, 626 (2013).

[34] J. A. Mundy, Y. Hikita, T. Hidaka, T. Yajima, T. Higuchi, H. Y. Hwang, D. A. Muller, and L. F. Kourkoutis, Nat. Commun. 5, 3464 (2014). 